VoL. 73 (2006) [345-351]

\title{
EXISTENCE OF TORUS BUNDLES ASSOCIATED TO COCYCLES
}

\author{
MiN Ho LeE
}

\begin{abstract}
A Kuga fibre variety is a fibre bundle over a locally symmetric space whose fibre is a polarized Abelian variety. We describe a complex torus bundle associated to a 2-cocycle of a discrete group, which may be regarded as a generalized Kuga fibre variety, and prove the existence of such a bundle.
\end{abstract}

\section{INTRODUCTION}

Families of Abelian varieties parametrised by an arithmetic quotient of a Hermitian symmetric domain play an important role in number theory. When such a family is viewed as a fibre bundle over an arithmetic variety its base space is essentially a Shimura variety and its total space is a Kuga fibre variety. This paper is concerned with a complex torus bundle associated to a 2-cocycle of a discrete group which may be regarded as a generalised Kuga fibre variety.

Let $\mathcal{H}_{n}$ be the Siegel upper half space of degree $n$ on which the symplectic group $S p(n, \mathbb{R})$ acts as usual. If $\Gamma^{\prime}$ is an arithmetic subgroup of $S p(n, \mathbb{R})$, then the associated quotient space $\Gamma^{\prime} \backslash \mathcal{H}_{n}$ can be regarded as a moduli space of a certain family of polarised Abelian varieties. Such a family of Abelian varieties can be considered as a fibre variety over the Siegel modular variety $X^{\prime}=\Gamma^{\prime} \backslash \mathcal{H}_{n}$.

Let $G=\mathbb{G}(\mathbb{R})$ be a semisimple Lie group of Hermitian type that can be realised as the set of real points of a linear algebraic group $\mathbb{G}$ defined over $\mathbb{Q}$. Thus the quotient $\mathcal{D}=G / K$ by a maximal compact subgroup $K$ of $G$ has the structure of a Hermitian symmetric domain. We assume that there are a holomorphic map $\tau: \mathcal{D} \rightarrow \mathcal{H}_{n}$ and a homomorphism $\rho: G \rightarrow S p(n, \mathbb{R})$ of Lie groups defined over $\mathbb{Q}$ such that $\tau$ is equivariant with respect to $\rho$, meaning that $\tau(g z)=\rho(g) \tau(z)$ for all $z \in \mathcal{D}$ and $g \in G$. Let $\Gamma$ be a torsion-free arithmetic subgroup of $G$ such that $\rho(\Gamma) \subset \Gamma^{\prime}$. Then the corresponding locally symmetric space $X=\Gamma \backslash \mathcal{D}$ has the structure of a complex variety and is called an arithmetic variety. The holomorphic map $\tau$ induces a morphism $\tau_{X}: X \rightarrow X^{\prime}$ of varieties, and by pulling the fibre variety over $X^{\prime}$ back via $\tau_{X}$ we obtain a fibre variety over $X$ whose fibres are again polarised Abelian varieties. Such fibre varieties over an arithmetic variety are called Kuga fibre varieties (see $[1,5]$ ), and various geometric and

Received 21st November, 2005

Copyright Clearance Centre, Inc. Serial-fee code: 0004-9727/06 \$A2.00+0.00. 
arithmetic aspects of Kuga fibre varieties have been investigated in numerous papers over the years.

If $L$ is a lattice in $\mathbb{R}^{2 n}$, whose elements are regarded as row vectors, satisfying $\ell \rho(\gamma)$ $\in L$ for all $\ell \in L$ and $\gamma \in \Gamma$, then the associated Kuga fibre variety can be considered as the quotient of $\mathcal{D} \times V$ by an action of the semidirect product $\Gamma \times L$. Given a 2-cocycle $\psi: \Gamma \times \Gamma \rightarrow L$, we can consider a generalised semidirect product $\Gamma \ltimes_{\psi} L$ by modifying its multiplication operation by using $\psi$. Then we can define an action of this generalised semidirect product on $\mathcal{D} \times V$ by using a 1-cochain $\xi$ for $\Gamma$ with coefficients in the space $\mathcal{A}\left(\mathcal{D}, \mathbb{C}^{n}\right)$ of $\mathbb{C}^{n}$-valued holomorphic functions which together with $\psi$ satisfies a certain relation (see Section 2), and the associated quotient is also a complex torus bundle over $\Gamma \backslash \mathcal{D}$. In [2] some properties of such torus bundles were investigated (see also [4] and [3, Chapter 8]). Since then, there have been inquiries about the existence of a pair $(\xi, \psi)$ satisfying the required condition. The purpose of this paper is to prove the existence of such a pair and provide an example.

\section{TORUS BUNDLES}

In this section we review the construction of a torus bundle associated to a 2-cocycle and a 1-cochain of a discrete subgroup of a semisimple Lie group considered in [2]. Let $G$ be a semisimple Lie group of Hermitian type, so that its quotient $\mathcal{D}=G / K$ by a maximal compact subgroup $K$ has the structure of a Hermitian symmetric domain. We assume that there are a holomorphic map $\tau: \mathcal{D} \rightarrow \mathcal{H}_{n}$ and a homomorphism $\rho: G \rightarrow S p(n, \mathbb{R})$ of Lie groups satisfying the equivariance condition

$$
\tau(g z)=\rho(g) \tau(z)
$$

for all $z \in \mathcal{D}$ and $g \in G$.

Let $L$ be a lattice in $\mathbb{R}^{2 n}$, and let $\Gamma$ be a discrete subgroup of $G$ such that $\ell \rho(\gamma) \in L$ for all $\ell \in L$ and $\gamma \in \Gamma$, where $\ell \rho(\gamma)$ is the matrix product of the row vector $\ell$ of $2 n$ entries and the $2 n \times 2 n$ matrix $\rho(\gamma)$. Then $L$ is a right $\Gamma$-module, and therefore we can consider the cohomology $H^{*}(\Gamma, L)$ of the group $\Gamma$ with coefficients in $L$. We denote by $\mathfrak{C}^{k}(\Gamma, L)$ and $3^{k}(\Gamma, L)$ the spaces of the associated $k$-cochains and $k$-cocycles, respectively, and choose an element $\psi$ of $3^{2}(\Gamma, L)$. Thus $\psi$ is a map $\psi: \Gamma \times \Gamma \rightarrow L$ satisfying

$$
\begin{gathered}
\psi\left(\gamma_{1}, \gamma_{2}\right) \rho\left(\gamma_{3}\right)+\psi\left(\gamma_{1} \gamma_{2}, \gamma_{3}\right)=\psi\left(\gamma_{2}, \gamma_{3}\right)+\psi\left(\gamma_{1}, \gamma_{2} \gamma_{3}\right) \\
\psi(\gamma, 1)=0=\psi(1, \gamma)
\end{gathered}
$$

for all $\gamma_{1}, \gamma_{2}, \gamma_{3}, \gamma \in \Gamma$, where 1 is the identity element of $\Gamma$. We note that an element $\alpha \in \mathfrak{Z}^{2}(\Gamma, L)$ is a coboundary if $\alpha=\partial \beta$ for some $\beta \in \mathfrak{C}^{1}(\Gamma, L)$, where

$$
\partial \beta\left(\gamma_{1}, \gamma_{2}\right)=\beta\left(\gamma_{2}\right)-\beta\left(\gamma_{1} \gamma_{2}\right)+\beta\left(\gamma_{1}\right) \rho\left(\gamma_{2}\right)
$$


for all $\gamma_{1}, \gamma_{2} \in \Gamma$. We now consider the generalised semidirect product $\Gamma \ltimes_{\psi} L$ associated to $\psi$, which consists of the elements $(\gamma, \ell)$ of $\Gamma \times L$ and is equipped with the multiplication operation defined by

$$
\left(\gamma_{1}, \ell_{1}\right) \cdot\left(\gamma_{2}, \ell_{2}\right)=\left(\gamma_{1} \gamma_{2}, \ell_{1} \rho\left(\gamma_{2}\right)+\ell_{2}+\psi\left(\gamma_{1}, \gamma_{2}\right)\right)
$$

for all $\gamma_{1}, \gamma_{2} \in \Gamma$ and $\ell_{1}, \ell_{2} \in L$. Then it can be shown that $\Gamma \propto_{\psi} L$ is a group with respect to this operation.

The symplectic group $S p(n, \mathbb{R})$ acts on the Siegel upper half space $\mathcal{H}_{n}$ as usual by

$$
g \zeta=(a \zeta+b)(c \zeta+d)^{-1}
$$

for all $z \in \mathcal{H}_{n}$ and $g=\left(\begin{array}{ll}a & b \\ c & d\end{array}\right) \in S p(n, \mathbb{R})$. For such $g \in S p(n, \mathbb{R})$ and $\zeta \in \mathcal{H}_{n}$, we set

$$
j(g, \zeta)=c \zeta+d .
$$

Then the resulting map $j: S p(n, \mathbb{R}) \times \mathcal{H}_{n} \rightarrow G L(n, \mathbb{C})$ satisfies

$$
j\left(g^{\prime} g, \zeta\right)=j\left(g^{\prime}, g \zeta\right) j(g, \zeta)
$$

for all $\zeta \in \mathcal{H}_{n}$ and $g, g^{\prime} \in S p(n, \mathbb{R})$. Given $z \in \mathcal{D}$ and $\gamma \in \Gamma \subset G$, we set

$$
j_{\rho, \tau}(\gamma, z)=j(\rho(\gamma), \tau(z)) .
$$

Using (2.4) and the fact that $\tau$ is an equivariant with respect to $\rho$, we see that

$$
j_{\rho, \tau}\left(\gamma^{\prime} \gamma, z\right)=j_{\rho, \tau}\left(\gamma^{\prime}, \gamma z\right) j_{\rho, \tau}(\gamma, z)
$$

for all $z \in \mathcal{D}$ and $\gamma, \gamma^{\prime} \in \Gamma$.

Let $\mathcal{A}\left(\mathcal{D}, \mathbb{C}^{n}\right)$ denote the space of $\mathbb{C}^{n}$-valued holomorphic functions on $\mathcal{D}$. We introduce on $\mathcal{A}\left(\mathcal{D}, \mathbb{C}^{n}\right)$ a double $\Gamma$-module structure defined by

$$
(\gamma \cdot f)(z)=f(z), \quad(f \cdot \gamma)(z)=f(\gamma z) j_{\rho, \tau}(\gamma, z)
$$

for all $f \in \mathcal{A}\left(\mathcal{D}, \mathbb{C}^{n}\right), \gamma \in \Gamma$ and $z \in \mathcal{D}$, where elements of $\mathbb{C}^{n}$ are considered as row vectors. Then we can consider the cohomology of the group $\Gamma$ with coefficients in $\mathcal{A}\left(\mathcal{D}, \mathbb{C}^{n}\right)$, where its group $\mathfrak{C}^{k}\left(\Gamma, \mathcal{A}\left(\mathcal{D}, \mathbb{C}^{n}\right)\right)$ of $k$-cochains consists of all functions

$$
\eta: \Gamma^{k} \rightarrow \mathcal{A}\left(\mathcal{D}, \mathbb{C}^{n}\right)
$$

such that $\eta\left(\gamma_{1}, \ldots, \gamma_{k}\right)=0$ whenever at least one of the $\gamma_{i}$ is 1 . If $\delta$ denotes the coboundary operator and if $\eta$ is a 1 -cochain belonging to $\mathbb{C}^{1}\left(\Gamma, \mathcal{A}\left(\mathcal{D}, \mathbb{C}^{n}\right)\right)$, then we have

$$
\delta \eta\left(\gamma_{1}, \gamma_{2}\right)=\gamma_{1} \cdot \eta\left(\gamma_{2}\right)-\eta\left(\gamma_{1} \gamma_{2}\right)+\eta\left(\gamma_{1}\right) \cdot \gamma_{2}
$$


for all $\gamma_{1}, \gamma_{2} \in \Gamma$, where the right and left actions of $\Gamma$ are given by (2.7).

We now assume that there exist a 1-cochain $\xi \in \mathbb{C}^{1}\left(\Gamma, \mathcal{A}\left(\mathcal{D}, \mathbb{C}^{n}\right)\right)$ and a 2-cocycle $\psi \in \mathfrak{Z}^{2}(\Gamma, L)$ satisfying

$$
\delta \xi\left(\gamma_{1}, \gamma_{2}\right)(z)=\psi\left(\gamma_{1}, \gamma_{2}\right)\left(\begin{array}{c}
\tau(z) \\
1
\end{array}\right)
$$

for all $z \in \mathcal{D}$, where the right hand side is the matrix product of the row vector $\psi\left(\gamma_{1}, \gamma_{2}\right)$ $\in L \subset \mathbb{R}^{n} \times \mathbb{R}^{n}$ and the complex $2 n \times n$ matrix $\left(\begin{array}{c}\tau(z) \\ 1\end{array}\right)$. The existence of such a pair $(\xi, \psi)$ is the main theorem in this paper and will be proved in the next section. Given elements $(\gamma,(\mu, \nu)) \in \Gamma \ltimes_{\psi} L$ and $(z, w) \in \mathcal{D} \times \mathbb{C}^{n}$, we set

$$
(\gamma,(\mu, \nu)) \cdot(z, w)=\left(\gamma z,(w+\mu \tau(z)+\nu+\xi(\gamma)(z)) j_{\rho, \tau}(\gamma, z)^{-1}\right),
$$

where $j_{\rho, \tau}: \Gamma \times \mathcal{D} \rightarrow G L(n, \mathbb{C})$ is given by $(2.5)$. Then it can be shown that the operation given by (2.10) determines an action of the group $\Gamma \ltimes_{\psi} L$ on the space $\mathcal{D} \times \mathbb{C}^{n}$.

We assume that the discrete subgroup $\Gamma \subset G$ does not contain elements of finite order, so that the quotient space $X=\Gamma \backslash \mathcal{D}$ has the structure of a complex manifold, and set

$$
Y_{\xi, \psi}=\Gamma \ltimes_{\psi} L \backslash \mathcal{D} \times \mathbb{C}^{n},
$$

where the quotient is taken with respect to the action in (2.10). Given $z \in \mathcal{D}$, let $L_{z}$ denote the lattice in $\mathbb{C}^{n}$ defined by

$$
L_{z}=\{\mu \tau(z)+\nu \mid(\mu, \nu) \in L\} .
$$

Then the map $\pi: Y_{\xi, \psi} \rightarrow X$ induced by the natural projections $\mathcal{D} \times \mathbb{C}^{n} \rightarrow \mathcal{D}$ and $\Gamma \alpha_{\psi} L \rightarrow \Gamma$ has the structure of a fibre bundle over $X$ whose fibre over the point in $X$ corresponding to $z \in \mathcal{D}$ is isomorphic to the complex torus $\mathbb{C}^{n} / L_{z}$. If $\psi=0$ and $\xi=0$, then the the corresponding torus bundle $Y_{0,0}$ is a family of Abelian varieties known as a Kuga fibre variety (see $[\mathbf{1}, \mathbf{5}]$ ).

\section{EXISTENCE}

Let the Hermitian symmetric domain $\mathcal{D}$ associated to a semisimple Lie group $G$, the discrete subgroup $\Gamma$ of $G$, and the lattice $L \subset \mathbb{C}^{n}$ be as in Section 2. In this section we prove the existence of a 1-cochain $\xi \in \mathfrak{C}^{1}\left(\Gamma, \mathcal{A}\left(\mathcal{D}, \mathbb{C}^{n}\right)\right)$ and a 2-cocycle $\psi \in \mathfrak{Z}^{2}(\Gamma, L)$ considered in Section 2 satisfying (2.9), where $\mathcal{A}\left(\mathcal{D}, \mathbb{C}^{n}\right)$ is the space of $\mathbb{C}^{n}$-valued holomorphic functions on $\mathcal{D}$. We also discuss an example.

We consider the space $\mathcal{A}\left(\mathcal{H}_{n}, \mathbb{C}^{n}\right)$ of $\mathbb{C}^{n}$-valued holomorphic functions on the Siegel upper half space $\mathcal{H}_{n}$ as a double $\Gamma$-module by defining the left and right $\Gamma$-actions by

$$
(g \cdot F)(\zeta)=F(\zeta), \quad(F \cdot g)(\zeta)=F(g \zeta) j(g, \zeta)
$$


for all $F \in \mathcal{A}\left(\mathcal{H}_{n}, \mathbb{C}^{n}\right), g \in S p(n, \mathbb{R})$ and $\zeta \in \mathcal{H}_{n}$, where $j(g, \zeta)$ is as in (2.3). Let $\widetilde{\Gamma}$ be a discrete subgroup of $S p(n, \mathbb{R})$ such that $\rho(\Gamma) \subset \widetilde{\Gamma}$. Then we can consider the cohomology of the group $\widetilde{\Gamma}$ with coefficients in $\mathcal{A}\left(\mathcal{H}_{n}, \mathbb{C}^{n}\right)$, and the coboundary operator

$$
\widetilde{\delta}: \mathfrak{C}^{1}\left(\widetilde{\Gamma}, \mathcal{A}\left(\mathcal{H}_{n}, \mathbb{C}^{n}\right)\right) \rightarrow \mathfrak{C}^{2}\left(\widetilde{\Gamma}, \mathcal{A}\left(\mathcal{H}_{n}, \mathbb{C}^{n}\right)\right)
$$

is given by

$$
\widetilde{\delta} \tilde{\eta}\left(g_{1}, g_{2}\right)=g_{1} \cdot \tilde{\eta}\left(g_{2}\right)-\tilde{\eta}\left(g_{1} g_{2}\right)+\tilde{\eta}\left(g_{1}\right) \cdot g_{2}
$$

for $\widetilde{\eta} \in \mathfrak{C}^{1}\left(\widetilde{\Gamma}, \mathcal{A}\left(\mathcal{H}_{n}, \mathbb{C}^{n}\right)\right)$ and $\gamma_{1}, \gamma_{2} \in \Gamma$, where the left and right actions of $\Gamma$ are given by $(3.1)$.

THEOREM 3.1. There exist a 1-cochain $\xi \in \mathfrak{C}^{1}\left(\Gamma, \mathcal{A}\left(\mathcal{D}, \mathbb{C}^{n}\right)\right)$ and a 2-cocycle $\psi \in \mathfrak{Z}^{2}(\Gamma, L)$ satisfying (2.9).

Proof: We shall first prove that there exist a 2-cocycle $\widetilde{\psi} \in \mathfrak{Z}^{2}(\widetilde{\Gamma}, L)$ and a 1-cochain $\tilde{\xi}$ belonging to $\mathfrak{C}^{1}\left(\widetilde{\Gamma}, \mathcal{A}\left(\mathcal{H}_{n}, \mathbb{C}^{n}\right)\right)$ satisfying

$$
\widetilde{\delta \xi}\left(g_{1}, g_{2}\right)(\zeta)=\tilde{\psi}\left(g_{1}, g_{2}\right)\left(\begin{array}{l}
\zeta \\
1
\end{array}\right)
$$

for all $\zeta \in \mathcal{H}_{n}$ and $g_{1}, g_{2} \in \widetilde{\Gamma}$. Let $\tilde{\xi}: \widetilde{\Gamma} \rightarrow \mathcal{A}\left(\mathcal{H}_{n}, \mathbb{C}^{n}\right)$ be an element of $\mathfrak{C}^{1}\left(\widetilde{\Gamma}, \mathcal{A}\left(\mathcal{H}_{n}, \mathbb{C}^{n}\right)\right)$ defined by

$$
\tilde{\xi}(g)(\zeta)=\kappa j(g, \zeta)
$$

for all $\zeta \in \mathcal{H}_{n}$ and $g \in \widetilde{\Gamma}$, where $\kappa \in \mathbb{C}$ is a constant and $j(g, \zeta)$ is as in (2.3). Then for this $\tilde{\xi}$ the condition (3.3) can be written as

$$
\begin{aligned}
\tilde{\psi}\left(g_{1}, g_{2}\right)\left(\begin{array}{l}
\zeta \\
1
\end{array}\right) & =\tilde{\xi}\left(g_{2}\right)(\zeta)-\tilde{\xi}\left(g_{1} g_{2}\right)(z)+\tilde{\xi}\left(g_{2}\right)\left(g_{2} \zeta\right) j\left(g_{2}, \zeta\right) \\
& =j\left(g_{2}, \zeta\right)-j\left(g_{1} g_{2}, \zeta\right)+j\left(g_{1}, g_{2} \zeta\right) j\left(g_{2}, \zeta\right)=j\left(g_{2}, \zeta\right)
\end{aligned}
$$

for all $\gamma_{1}, \gamma_{2} \in \Gamma$ and $z \in \mathcal{D}$, where we used (2.4) and (3.2). Here we note that it is possible choose a map $\psi: \Gamma \times \Gamma \rightarrow V$ satisfying (3.4). For example, if $\tilde{\psi}$ is given by

$$
\tilde{\psi}\left(g_{1}, g_{2}\right)=\left((0,1) g_{2}\left(\begin{array}{l}
1 \\
0
\end{array}\right),(0,1) g_{2}\left(\begin{array}{l}
1 \\
0
\end{array}\right)\right)
$$

then for $g_{2}=\left(\begin{array}{ll}a & b \\ c & d\end{array}\right)$ we have

$$
\tilde{\psi}\left(g_{1}, g_{2}\right)\left(\begin{array}{l}
\zeta \\
1
\end{array}\right)=(c, d)\left(\begin{array}{l}
\zeta \\
1
\end{array}\right)=c \zeta+d=j\left(g_{2}, \zeta\right) .
$$


Let $\tilde{\psi}: \widetilde{\Gamma} \times \widetilde{\Gamma} \rightarrow L$ be a map satisfying (3.4). Using (2.1) and (2.4), we have

$$
\begin{aligned}
\left(\tilde{\psi}\left(g, g^{\prime}\right) g^{\prime \prime}\right. & \left.+\widetilde{\psi}\left(g g^{\prime}, g^{\prime \prime}\right)-\widetilde{\psi}\left(g^{\prime}, g^{\prime \prime}\right)-\tilde{\psi}\left(g, g^{\prime} g^{\prime \prime}\right)\right)\left(\begin{array}{l}
\zeta \\
1
\end{array}\right) \\
& =\widetilde{\psi}\left(g g^{\prime}\right)\left(\begin{array}{c}
g^{\prime \prime} \zeta \\
1
\end{array}\right) j\left(g^{\prime \prime}, \zeta\right)+j\left(g^{\prime \prime}, \zeta\right)-j\left(g^{\prime \prime}, \zeta\right)-j\left(g^{\prime} g^{\prime \prime}, \zeta\right) \\
& =j\left(g^{\prime}, g^{\prime \prime} \zeta\right) j\left(g^{\prime \prime}, \zeta\right)-j\left(g^{\prime} g^{\prime \prime}, \zeta\right)=0
\end{aligned}
$$

for $\zeta \in \mathcal{H}_{n}$ and $g, g^{\prime}, g^{\prime \prime} \in \Gamma$. This implies that $\tilde{\psi}$ satisfies $(2.1)$; hence $\tilde{\psi} \in \mathfrak{Z}^{2}(\widetilde{\Gamma}, L)$. We now set

$$
\psi\left(\gamma_{1}, \gamma_{2}\right)=\widetilde{\psi}\left(\rho\left(\gamma_{1}\right), \rho\left(\gamma_{2}\right)\right), \quad \xi(\gamma)(z)=\tilde{\xi}(\rho(\gamma))(\tau(z))
$$

for all $\gamma, \gamma_{1}, \gamma_{2} \in \Gamma$ and $z \in \mathcal{D}$. Then $\psi \in \mathcal{Z}^{2}(\Gamma, L)$ and $\xi \in \mathbb{C}^{1}\left(\Gamma, \mathcal{A}\left(\mathcal{D}, \mathbb{C}^{n}\right)\right)$, and by using (2.2) and (3.2) as well as (3.3) we obtain

$$
\begin{aligned}
\delta \xi\left(\gamma_{1}, \gamma_{2}\right)(z)= & \xi\left(\gamma_{2}\right)(z)-\xi\left(\gamma_{1} \gamma_{2}\right)(z)+\xi\left(\gamma_{2}\right)\left(\gamma_{2} z\right) j_{\rho, \tau}\left(\gamma_{2}, z\right) \\
= & \widetilde{\xi}\left(\rho\left(\gamma_{2}\right)\right)(\tau(z))-\widetilde{\xi}\left(\rho\left(\gamma_{1}\right) \rho\left(\gamma_{2}\right)\right)(\tau(z)) \\
& +\widetilde{\xi}\left(\rho\left(\gamma_{2}\right)\right)\left(\tau\left(\gamma_{2} z\right)\right) j\left(\rho\left(\gamma_{2}\right), \tau(z)\right) \\
& =\widetilde{\delta \xi}\left(\rho\left(\gamma_{1}\right), \rho\left(\gamma_{2}\right)\right)(\tau(z)) \\
& =\widetilde{\psi}\left(\rho\left(\gamma_{1}\right), \rho\left(\gamma_{2}\right)\right)\left(\begin{array}{c}
\tau(z) \\
1
\end{array}\right)=\psi\left(\gamma_{1}, \gamma_{2}\right)\left(\begin{array}{c}
\tau(z) \\
1
\end{array}\right) .
\end{aligned}
$$

Thus we see that the pair $(\xi, \psi)$ satisfies $(2.9)$, and therefore the proof of the theorem is complete.

Example 3.2. Let $\xi_{0} \in \mathfrak{C}^{1}\left(\Gamma, \mathcal{A}\left(\mathcal{D}, \mathbb{C}^{n}\right)\right)$ be the 1-cochain given by

$$
\xi_{0}(\gamma)(z)=\kappa j_{\rho, \tau}(\gamma, z)
$$

for all $\gamma \in \Gamma$ and $z \in \mathcal{D}$, where $\kappa \in \mathbb{C}$ is a constant and $j_{\rho, \tau}(\gamma, z)$ is as in (2.5). If $\psi_{0} \in \mathfrak{Z}^{2}(\Gamma, L)$ is the 2 -cocycle given by

$$
\psi_{0}\left(\gamma_{1}, \gamma_{2}\right)=\left((0,1) \rho\left(\gamma_{2}\right)\left(\begin{array}{l}
1 \\
0
\end{array}\right),(0,1) \rho\left(\gamma_{2}\right)\left(\begin{array}{l}
1 \\
0
\end{array}\right)\right)
$$

for all $\gamma_{1}, \gamma_{2} \in \Gamma$, then from the proof of Theorem 3.1 we see that the pair $\left(\xi_{0}, \psi_{0}\right)$ satisfies (2.9). By using this pair we can construct other pairs as follows. Let $\varepsilon$ be a 1-cochain belonging to $\mathbb{C}^{1}(\Gamma, L)$, and consider the elements $\xi_{\varepsilon} \in \mathfrak{C}^{1}\left(\Gamma, \mathcal{A}\left(\mathcal{D}, \mathbb{C}^{n}\right)\right)$ and $\psi_{\varepsilon} \in \mathfrak{Z}^{2}(\Gamma, L)$ given by

$$
\xi_{\varepsilon}(\gamma)(z)=\xi_{0}(\gamma)(z)+\varepsilon(\gamma)\left(\begin{array}{c}
\tau(z) \\
1
\end{array}\right), \quad \psi_{\varepsilon}=\psi+\partial \varepsilon
$$


for all $\gamma \in \Gamma$ and $z \in \mathcal{D}$, where $\partial: \mathfrak{C}^{1}(\Gamma, L) \rightarrow \mathfrak{C}^{2}(\Gamma, L)$ is the coboundary operator. Then for $\gamma_{1}, \gamma_{2} \in \Gamma$ and $z \in \mathcal{D}$ we have

$$
\begin{aligned}
\partial \xi_{\varepsilon}\left(\gamma_{1}, \gamma_{2}\right)(z)= & \xi_{\varepsilon}\left(\gamma_{2}\right)(z)-\xi_{\varepsilon}\left(\gamma_{1} \gamma_{2}\right)(z)+\xi_{\varepsilon}\left(\gamma_{1}\right)\left(\gamma_{2} z\right) j_{\rho, \tau}\left(\gamma_{2}, z\right) \\
= & \partial \xi\left(\gamma_{1}, \gamma_{2}\right)(z)+\varepsilon\left(\gamma_{2}\right)\left(\begin{array}{c}
\tau(z) \\
1
\end{array}\right) \\
& \quad-\varepsilon\left(\gamma_{1} \gamma_{2}\right)\left(\begin{array}{c}
\tau(z) \\
1
\end{array}\right)+\varepsilon\left(\gamma_{1}\right)\left(\begin{array}{c}
\tau\left(\gamma_{2} z\right) \\
1
\end{array}\right) j_{\rho, \tau}\left(\gamma_{2}, z\right)
\end{aligned}
$$

However, we have

$$
\begin{aligned}
\left(\begin{array}{c}
\tau\left(\gamma_{2} z\right) \\
1
\end{array}\right) j_{\rho, \tau}\left(\gamma_{2}, z\right) & =\left(\begin{array}{c}
(a \tau(z)+b)(c \tau(z)+d)^{-1} \\
1
\end{array}\right)(c \tau(z)+d) \\
& =\left(\begin{array}{c}
a \tau(z)+b \\
c \tau(z)+d
\end{array}\right)=\rho\left(\gamma_{2}\right)\left(\begin{array}{c}
\tau(z) \\
1
\end{array}\right)
\end{aligned}
$$

if $\rho\left(\gamma_{2}\right)=\left(\begin{array}{ll}a & b \\ c & d\end{array}\right)$. Using this, (2.2), and (3.5), we see that

$$
\partial \xi_{\varepsilon}\left(\gamma_{1}, \gamma_{2}\right)(z)=\psi\left(\gamma_{1}, \gamma_{2}\right)\left(\begin{array}{c}
\tau(z) \\
1
\end{array}\right)+(\partial \varepsilon)\left(\gamma_{1}, \gamma_{2}\right)\left(\begin{array}{c}
\tau(z) \\
1
\end{array}\right)=\psi_{\varepsilon}\left(\gamma_{1}, \gamma_{2}\right)\left(\begin{array}{c}
\tau(z) \\
1
\end{array}\right)
$$

Thus the pair $\left(\xi_{\varepsilon}, \psi_{\varepsilon}\right)$ satisfies $(2.9)$.

\section{REFERENCES}

[1] M. Kuga, Fiber varieties over a symmetric space whose fibers are abelian varieties I, II (Univ. of Chicago, Chicago, 1963/64).

[2] M.H. Lee, 'Cohomology of complex torus bundles associated to cocycles', Canad. J. Math. 55 (2003), 839-855.

[3] M.H. Lee, Mixed automorphic forms, torus bundles, and Jacobi forms, Lecture Notes in Math. 1845 (Springer-Verlag, Berlin, 2004).

[4] M.H. Lee and D.Y. Suh, 'Torus bundles over locally symmetric varieties associated to cocycles of discrete groups', Monatsh. Math. 59 (2000), 127-141.

[5] I. Satake, Algebraic structures of symmetric domains (Princeton Univ. Press, Princeton, 1980).

Department of Mathematics University of Northern Iowa Cedar Falls, IA 50614

United States of America

e-mail: lee@math.uni.edu 